\title{
Subungual Solitary Angiokeratoma Simulating a Squamous Cell Carcinoma
}

\author{
Nélia Cunha ${ }^{a}$ Josette André ${ }^{b} \quad$ Ursula Sass $^{c}$ Bertrand Richert $^{b}$ \\ ${ }^{a}$ Dermatology Department, Hospital de Santo António dos Capuchos - Centro Hospitalar de Lisboa Central, \\ Lisbon, Portugal; ' ${ }^{b}$ Dermatology Department, CHU Brugmann and CHU Saint-Pierre, Université Libre de Bruxelles, \\ and ${ }^{C}$ Dermatology Department, CHU Saint-Pierre, Université Libre de Bruxelles, Brussels, Belgium
}

\section{Established Facts}

- Subungual solitary angiokeratoma is extremely rare.

- Only 1 case is reported in the literature, presenting as a longitudinal pigmented nail band.

\section{Novel Insights}

- We report a case of subungual solitary angiokeratoma clinically simulating a squamous cell carcinoma.

- Solitary angiokeratoma should be added to the differential diagnosis of subungual warty tumors.

\section{Keywords}

Angiokeratoma $\cdot$ Subungual $\cdot$ Nail tumor $\cdot$ Squamous cell carcinoma

\begin{abstract}
Subungual angiokeratoma is extremely rare. Only 1 case is reported in the literature, presenting as a longitudinal pigmented band on a toenail. We report a case of a subungual angiokeratoma on a fingernail of a 43-year-old woman, clinically mimicking a squamous cell carcinoma. Medical imaging revealed a soft tissue tumor and erosion of the distal phalanx. Although extremely rare, solitary angiokeratomas may arise in the nail apparatus and should be added to the differential diagnosis of subungual warty tumors.
\end{abstract}

(C) 2017 S. Karger AG, Basel

\section{KARGER}

(c) 2017 S. Karger AG, Basel

E-Mail karger@karger.com

www.karger.com/sad

\section{Introduction}

Angiokeratomas are benign vascular lesions characterized by dilated papillary dermal vessels and reactive hyperkeratosis. Five variants are currently recognized: solitary/multiple angiokeratomas, angiokeratomas of the scrotum and vulva, angiokeratoma of Mibelli, angiokeratoma corporis diffusum, and angiokeratoma circumscriptum [1]. Solitary angiokeratomas may present anywhere on the body, but its occurrence in the nail apparatus is exceptional, with only 1 case described in the literature [2]. Herein, we report a case of a subungual angiokeratoma on a fingernail, clinically mimicking squamous cell carcinoma (SCC) of the nail bed. 


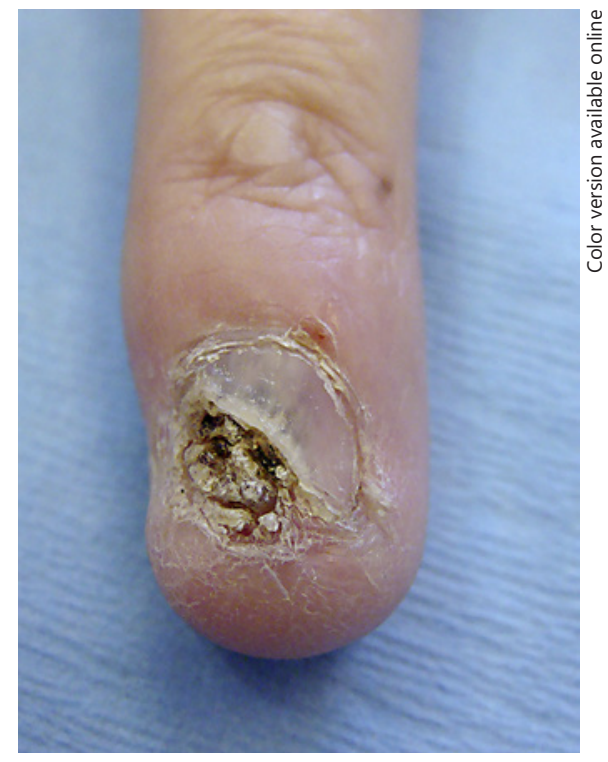

Fig. 1. Clipping of the onycholytic distorted nail plate exposed a violaceous warty lesion of the nail bed with hyperkeratosis and crusts.

\section{Case Report}

A 43-year-old woman presented with a history of onycholysis, subungual hyperkeratosis, and pain on the left fourth fingernail evolving for several years. The diagnoses of onychomycosis and wart had been considered by other physicians. She recalled an acute episode of spontaneous subungual purulent discharge resulting in pain relief and denied any particular local trauma. Her background history was remarkable for a previous heart transplant 10 years before, and she had been taking cyclosporin A since then. She is currently awaiting kidney transplantation due to renal insufficiency.

On clinical examination, the nail plate was distorted and elevated by a large subungual keratosis. Clipping of the onycholytic nail plate exposed a violaceous warty lesion of the nail bed with hyperkeratosis and crusts (Fig. 1), evoking a possible SCC of the nail bed. Radiography and magnetic resonance imaging of the finger were performed, revealing a soft tissue tumor of approximately $1 \mathrm{~cm}$ length and $5 \mathrm{~mm}$ thickness associated with an underlying subtle erosion of the distal phalanx (Fig. 2).

Under local anesthesia, the plate was laterally avulsed and the lesion removed, down to the bone, without any safety margin. The undersurface of the nail plate was scrapped away to collect any residual tumor. The plate was led back into its original position and sutured to the lateral nail fold (Fig. 3). Histopathological examination revealed irregular acanthosis, a thickened and parakeratotic stratum corneum filled with vesicles, and marked dilation of the papillary dermal vessels, which were surrounded by the epidermal rete ridges. These findings were consistent with an angiokeratoma (Fig. 4).

Following surgery, the nail growth was normal. At the 10-month follow-up, ill-defined purplish subungual macules, slight distal onycholysis, and hyperkeratosis were noticeable (Fig. 5). The diagno-

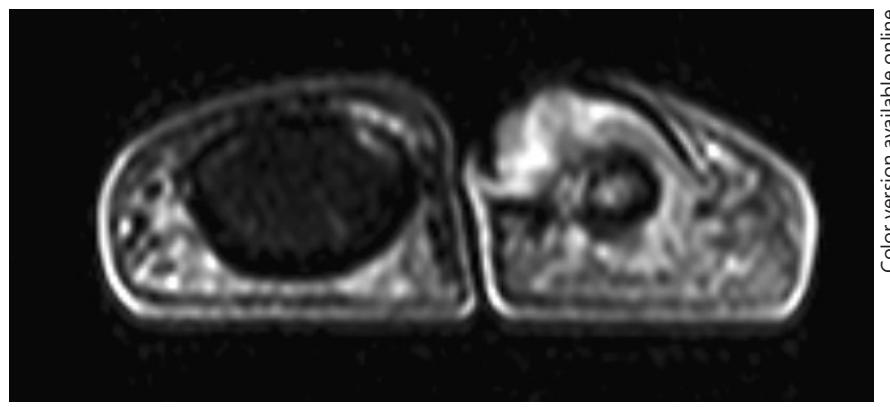

Fig. 2. MRI of the involved finger (on the right) and the adjacent middle finger. The tumor extends deeply in the lateral sulcus. Note that the signal is not homogeneous within the tumor.

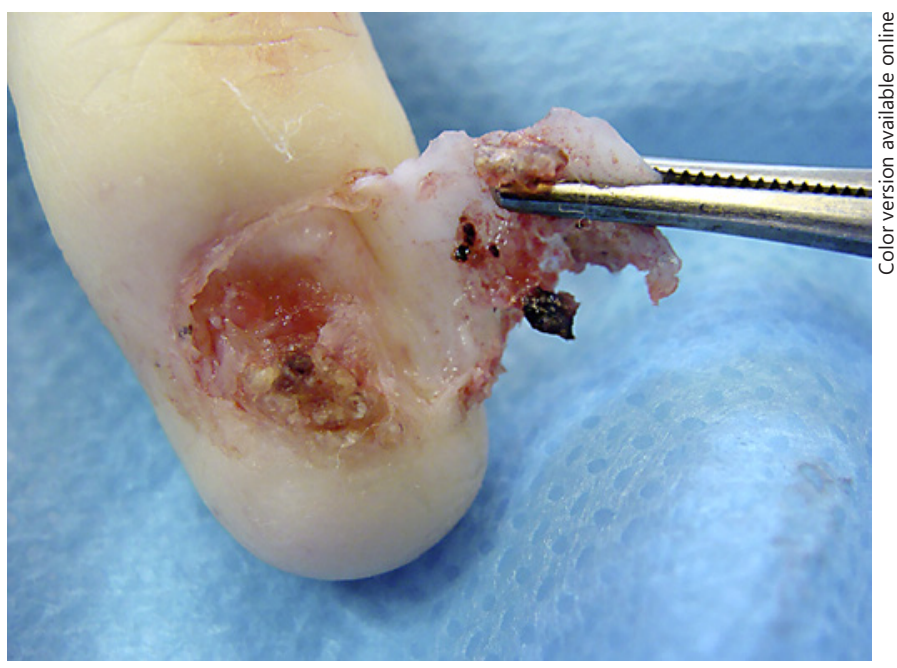

Fig. 3. Perioperative view: lateral nail avulsion exposes a keratotic tumor involving the whole lateral two-thirds of the nail bed. The proximal part of the tumor remained attached to the undersurface of the plate.

sis of recurrent angiokeratoma was made based on the clinical and dermoscopic findings and the previous history. Considering the benign nature of the lesion and the absence of pain, the patient declined further suggested treatments (pulsed dye laser and surgery) as she was mostly concerned with her pending renal transplant.

\section{Discussion}

Solitary angiokeratoma usually presents on nonglabrous skin, most commonly on the lower extremities, as a warty, slightly hyperkeratotic black papule that may pose differential diagnosis with melanoma, especially in case of thrombosis [1]. It has also been described on the tongue [3] and the palms and soles [4]. Its occurrence in 


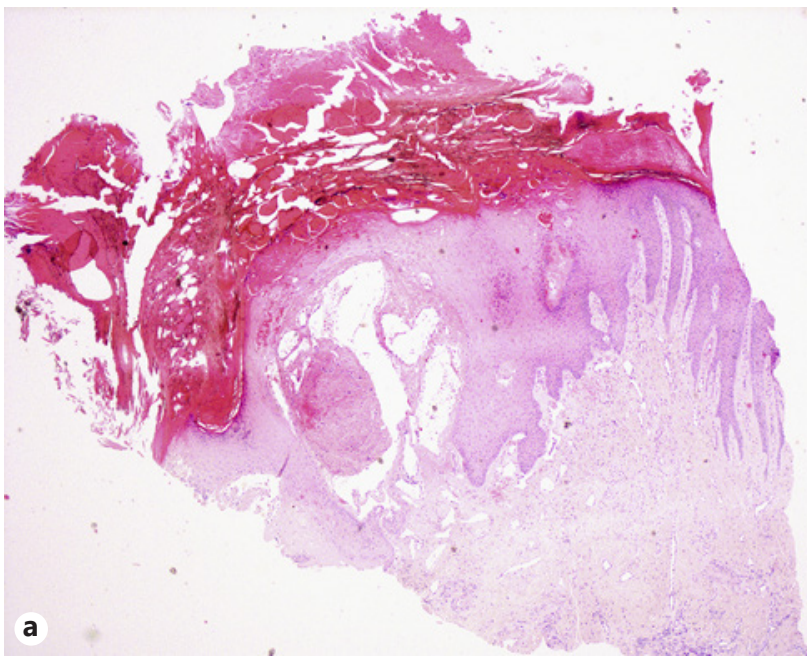

Fig. 4. Histology of the excised lesion. a There is irregular acanthosis and the stratum corneum is thickened, parakeratotic, and filled with vesicles. There is marked dilation of the papillary dermal vessels, which are surrounded by the epidermal rete ridges. Hematoxylin and eosin stain. Original magnification $\times 25$. b Dilated capillaries surrounded by epidermal rete ridges. Note a thrombus on the upper left. Hematoxylin and eosin stain. Original magnification $\times 100$. $c$ The capillaries are lined by a single layer of plump endothelial cells. Hematoxylin and eosin stain. Original magnification $\times 200$.

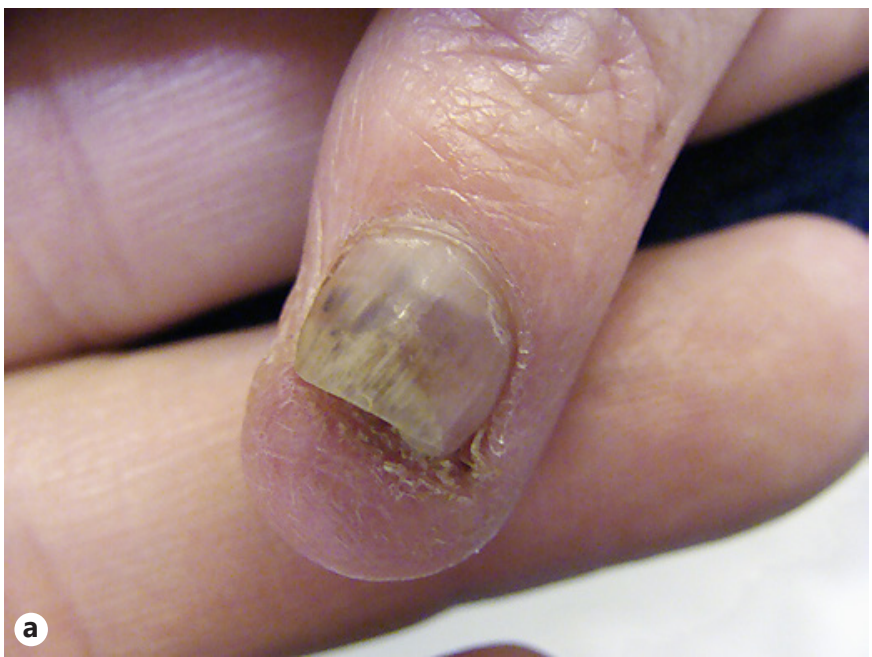

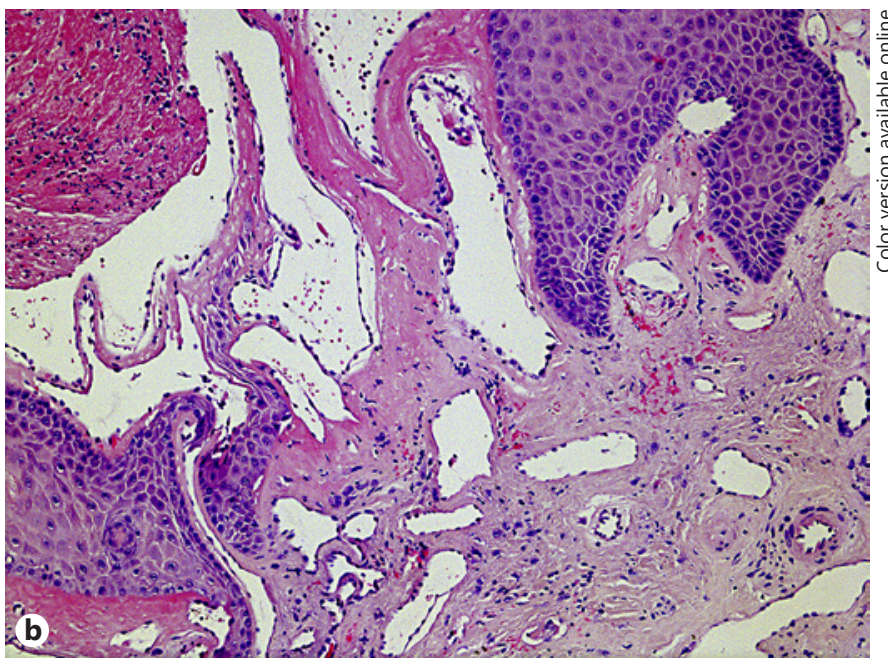
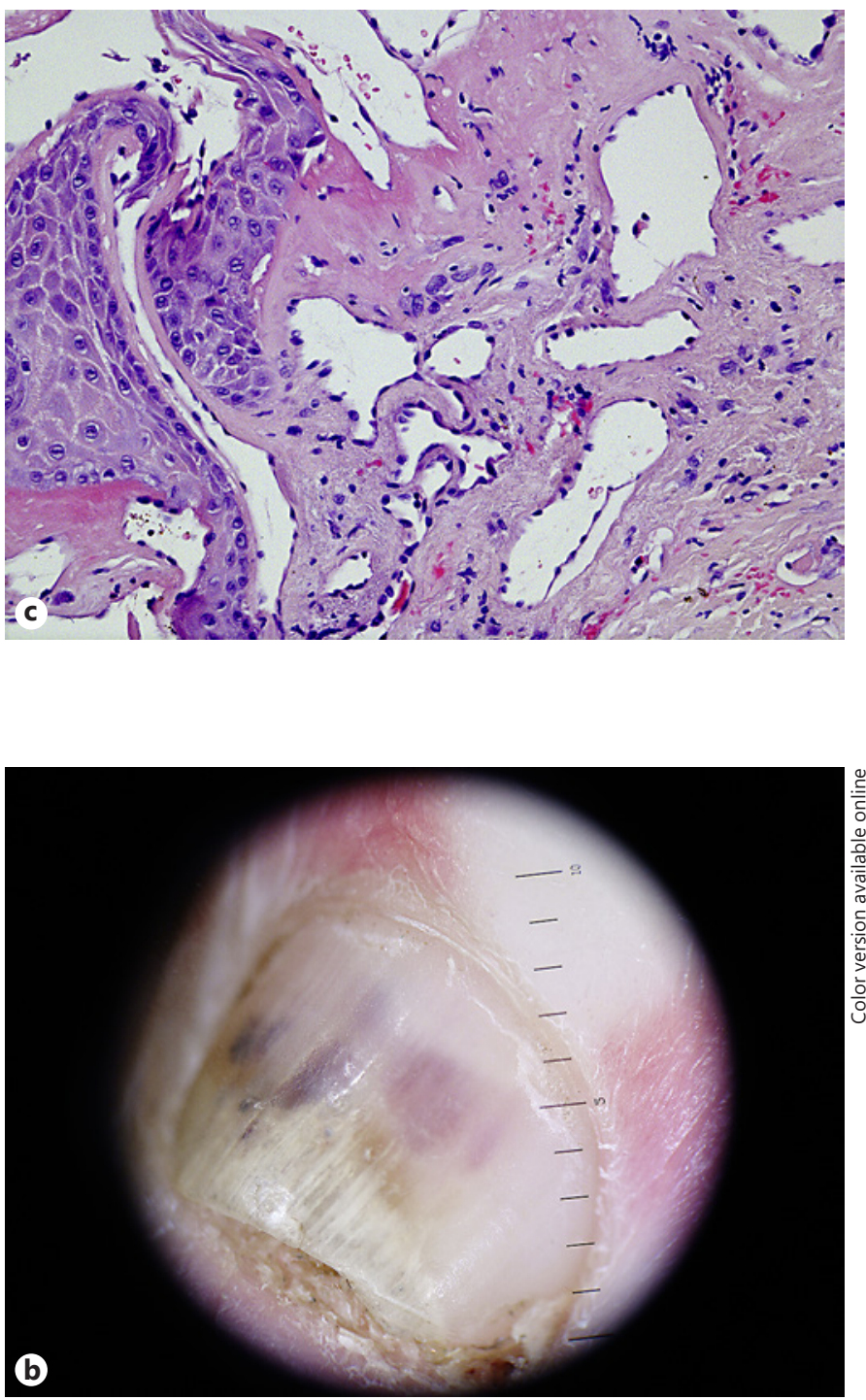

Fig. 5. Recurrence at the 10-month follow-up: clinical (a) and dermoscopic (b) findings. Ill-defined purplish subungual macules are noticeable through the nail plate associated with distal onycholysis. 
the nail apparatus was reported only once, in 2015, presenting as a longitudinal pigmented band on the left fourth toenail [2]. Our case is the second report of a subungual angiokeratoma, and the first on a fingernail, clinically mimicking a SCC of the nail unit.

SCC is the most frequent malignant tumor of the nail unit [5]. It affects mainly fingers and has a characteristic indolent course, with various possible presentations, leading to misdiagnosis as a wart or other benign conditions $[6,7]$. Subungual involvement and presentation as a warty lesion, with hyperkeratosis and onycholysis, have been found to be the most common findings [8]. Therefore, in our patient, the differential diagnosis with a SCC was crucial, even though SCC occurs mainly in men in their sixties and is usually painless [6-8]. Distal digit keratoacanthoma can also present with similar clinical findings but is usually very painful and has a characteristic rapid growth, causing a crescent-shaped osteolysis [9, $10]$.

Solitary angiokeratomas are thought to result from trauma or chronic irritation of a venule wall in the papillary dermis [1]. There is also 1 report of angiokeratomas appearing on the injection sites of etanercept but not on phlebotomy sites; the authors concluded that etanercept itself could have played a role [11]. In our case, no trau- matic origin could be established. The possible role of immunosuppression linked with the heart transplant is not probable either, as there are no reports of angiokeratoma growing incidence in immunosuppressed patients.

In conclusion, although extremely rare, solitary angiokeratomas may arise in the nail apparatus and may share similar clinical features with subungual warty SCC of the nail bed. Subungual angiokeratoma should be added to the differential diagnosis of warty subungual tumors.

\section{Acknowledgments}

Nélia Cunha acknowledges the Cabral de Ascensão Grant of the Portuguese Society of Dermatology and Venereology for fellowship support.

\section{Statement of Ethics}

The subject has given her oral informed consent for the photos and details of her case to be published.

\section{Disclosure Statement}

The authors have no conflict of interest to declare.

\section{References}

1 North PE, Kincannon J: Vascular neoplasms and neoplastic-like proliferations; in Bolognia JL, Jorizzo JL, Schaffer JV (eds): Dermatology. Elsevier Saunders, 2012, vol 2, pp 1915-1942.

2 Hasegawa M, Tamura A: Subungual angiokeratoma presenting as a longitudinal pigmented band in the nail. Acta Derm Venereol 2015;95:1001-1002.

3 Fernández-Aceñero MJ, Rey Biel J, Renedo G: Solitary angiokeratoma of the tongue in adults. Rom J Morphol Embryol 2010;51: 771-773.
4 Wang L, Gao T, Wang G: Solitary angiokeratoma on palms and soles: a clinicopathological analysis of 21 cases. J Dermatol 2013;40: 653-656.

5 Richert B, Lecerf P, Caucanas M, André J: Nail tumors. Clin Dermatol 2013;31:602-617.

6 Mikhail GR: Subungual epidermoid carcinoma. J Am Acad Dermatol 1984;11:291-298.

7 Dalle S, Depape L, Phan A, Balme B, RongerSavle S, Thomas L: Squamous cell carcinoma of the nail apparatus: clinicopathological study of 35 cases. Br J Dermatol 2007;156: 871-874.

8 Lecerf P, Richert B, Theunis A, André J: A retrospective study of squamous cell carcinoma of the nail unit diagnosed in a Belgian general hospital over a 15-year period. J Am Acad Dermatol 2013;69:253-261.
9 Baran R, Goettmann S: Distal digital keratoacanthoma: a report of 12 cases and a review of the literature. Br J Dermatol 1998;139:512515.

10 Levy DW, Bonakdarpour A, Putong PB, Mesgarzadeh M, Betz RR: Subungual keratoacanthoma. Skeletal Radiol 1985;13:287-290.

11 Bathelier E, Gunera-Saad N, Bérard F, Nicolas JF: Angiokeratoma induced by injection of etanercept for psoriasis. J Am Acad Dermatol 2008;59:S124-S125. 